\title{
What Happens after Kasai for Biliary Atresia? A European Multicenter Survey
}

\author{
Zeng Hao Wong ${ }^{1}$ Mark Davenport ${ }^{1}$ \\ ${ }^{1}$ Department of Paediatric Surgery, King's College Hospital, London, \\ United Kingdom \\ Eur J Pediatr Surg 2019;29:1-6.
}

Address for correspondence Mark Davenport, ChM, FRCS, Department of Paediatric Surgery, King's College Hospital, Denmark Hill, London SE5 9RS, United Kingdom (e-mail: markdav2@ntlworld.com).

\begin{abstract}
Keywords

- biliary atresia

- adjuvant therapy

- Kasai portoenterostomy

- corticosteroids

- ursodeoxycholic acid

Aim Biliary atresia (BA) remains a rare disease in Europe with an estimated incidence of 1 in 15-20,000 livebirths. While the Kasai portoenterostomy (KPE) is regarded as the standard operation for BA, there is no consensus on optimum adjuvant therapy. We sought to determine the variation in therapy and opinion in centers known to have an interest in pediatric hepatobiliary surgery across Europe.

Methods A survey of current pre- and postoperative practice was circulated to surgeons and centers known to have an interest in BA. Data are quoted as median (range).

Results There were 19 completed center forms from 12 different countries. Annual (new) caseload varied with 10 centers reported seeing $\leq 5$ patients/year and 4 centers $>15$ patients/year. The distribution of BA variants was isolated BA (80 [60-100]\%), syndromic BA (10 [3-35]\%), cystic BA (5 [1-15]\%), and cytomegalovirus (CMV) immunoglobulin M (IgM)-positive BA (5 [0-15]\% (CMV serology only formally tested in 16 centers). The commonest age group at KPE was 51 to 60 days $(n=8)$. All centers performed an exclusively open KPE, although one used laparoscopy for diagnosis. A steroid-based postoperative regimen was used in 11/19 (58\%) centers, but with marked variation in dose and duration. Commonest perioperative antibiotics were a combination of piperacillin-tazocin $(n=7)$ and gentamicin $(n=8)$. Oral prophylactic antibiotics, with varying duration (4-52 weeks) were used in 13/19 (68\%) centers. If CMV serology was positive, seven centers would treat with specific antiviral therapy. Other postoperative medication included ursodeoxycholic acid (UDCA) $(n=19)$, phenobarbitone $(n=4)$, and cholestyramine $(n=2)$. Self-declared clearance of jaundice in centers varied from 30 to $40 \%(n=3)$ to $>60 \%(n=5)$.

Conclusion All surveyed European centers continue to perform an exclusively open $\mathrm{KPE}$, but there is no consensus on a standard adjuvant drug regimen. Self-declared outcome post-KPE also appears to be variable though the reasons are obscure.
\end{abstract}

\section{Introduction}

Biliary atresia (BA) is a rare congenital obliterative cholangiopathy of the newborn. There is a marked geographical variation in incidence with higher reported rates in Asian countries, such as in Taiwan ( 1 in 5000 births) ${ }^{1}$ and Japan ( 1 in 8000$)^{2}$ By contrast, the reported rates in Europe are consistently lower ${ }^{3-6}$ and range from 1 in 14,000 (in Sweden)

received

May 15, 2018

accepted

June 26, 2018

published online

August 21, 2018

to approximately 1 in 19,000 in France. We have described at least four separate variants of BA with different etiological origins and clinical features including isolated $B A$, syndromic $\mathrm{BA}$, cystic BA, and most recently cytomegalovirus (CMV) immunoglobulin M positive (IgM + ve) BA. ${ }^{7}$

Most infants without clinical evidence of end-stage liver disease would be offered an attempt at restoration of bile

(c) 2019 Georg Thieme Verlag KG Stuttgart · New York
DOI https://doi.org/ 10.1055/s-0038-1668146. ISSN 0939-7248. 
flow and preservation of the native liver by a Kasai portoenterostomy (KPE). Although this approach would be regarded as standard throughout the world, there is considerable variation in the perceived efficacy and role of any other adjuvant pharmacological therapy.

The aim of this study was to investigate variation in perioperative management across Europe.

\section{Methods}

A standard questionnaire (- Supplementary Material, available in the online version) was designed focusing on possible perioperative regimens including steroids, ursodeoxycholic acid (UDCA), antibiotics, etc. Diagnostic methods, type of operation, and self-reported assessment of annual caseload and outcome were also ascertained.

The questionnaire was circulated to surgeons in European centers known to treat BA. When there were multiple respondents from a single center, a consensus or the most senior respondent's answers were taken. Data were quoted as median (range).

\section{Results}

\section{Centers}

This was a European multicenter $(n=19)$ study across 12 countries ( - Fig. 1 ), with 1 to $>15$ new patients treated per year. Ten centers reported seeing $\leq 5$ patients/year and four centers $>15$ patients/year.
Eighty (60-100) percent of infants were described as isolated BA, 10 (3-35) \% syndromic BA, 5 (1-15) \% cystic $\mathrm{BA}$, and 5 (0-15) \% CMV-positive BA.

Preoperative ultrasound was universal with others performed including percutaneous liver biopsy $(n=9$; radioisotope liver scan [ $n=5]$, magnetic resonance cholangiopancreatography [MRCP] $[n=3]$, endoscopic retrograde cholangiopancreatography [ERCP] $[n=1]$, and laparoscopic cholangiogram [ $n=1]$ ). A viral serology panel was tested in 16 centers ( - Fig. 2). The modal age group at KPE was 51 to 60 $(n=8)$ days, followed by $61-70(n=6)$ days old. Two smaller centers ( $<5 /$ year) in Germany and Turkey suggested that their average was $>70$ days.

All centers performed an open KPE, with some variation in technique. Thus, three different variations could be observed: original description according to Kasai with transection margin resembling "ovoid" remnant $(n=2)$, radical resection to limits circumscribed to margins of bifurcation of portal plate $(n=7)$, and radical resection involving dissection between anterior and posterior right vascular pedicles (Rouviere's fossa) to dissection within Rex fossa on the left side $(n=11)$. One center also resected part of segment $4 \mathrm{~b}$ as a routine.

\section{Adjuvant Therapy}

\section{Steroids}

Steroids were used in 11/19 (58\%) centers although with a marked variation in regimens. The commonest was oral prednisolone/prednisone $(n=8)$, which was preceded by

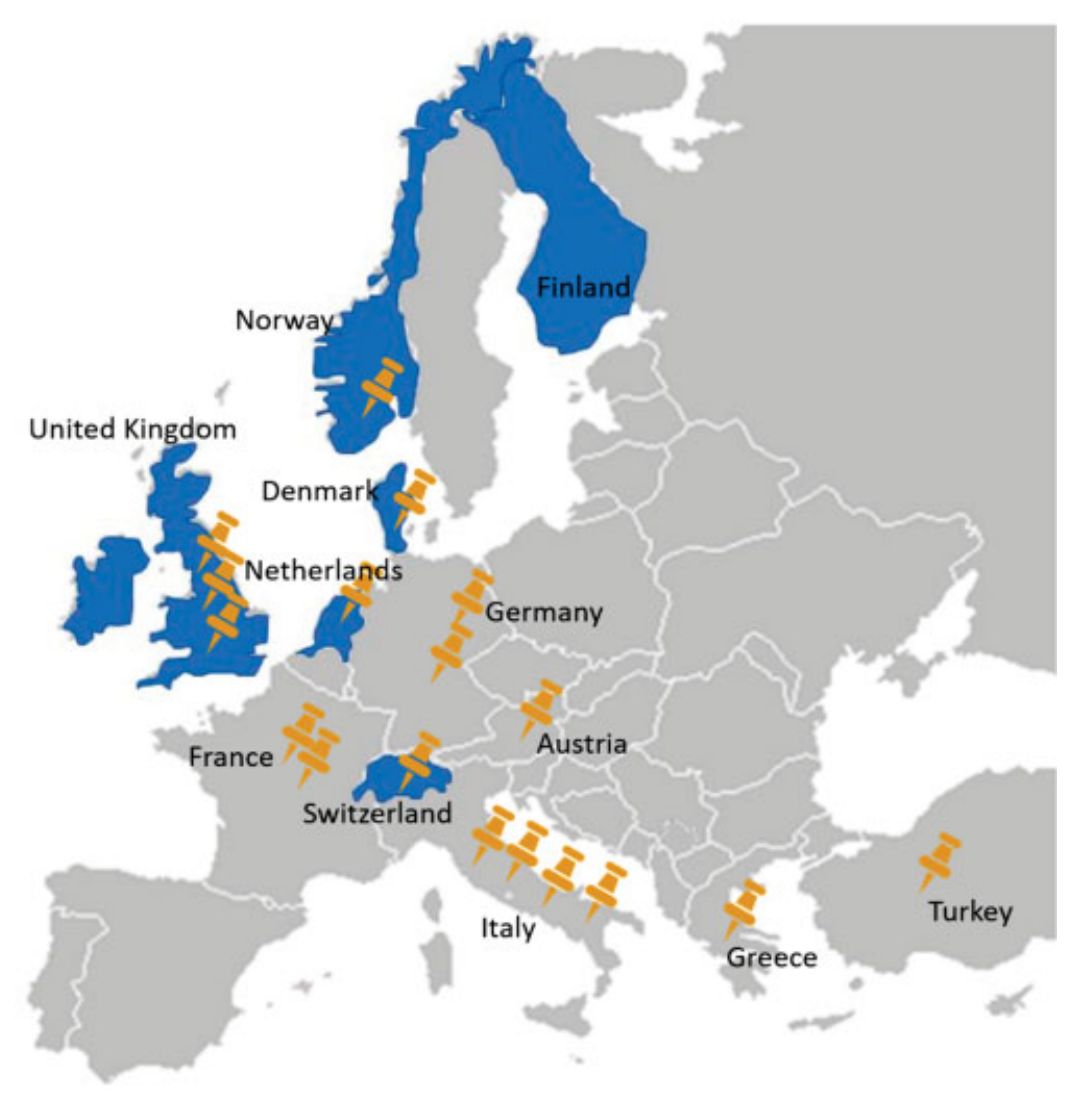

Fig. 1 Respondent centers (thumbnails). Countries with national centralization of biliary atresia management (in blue). 


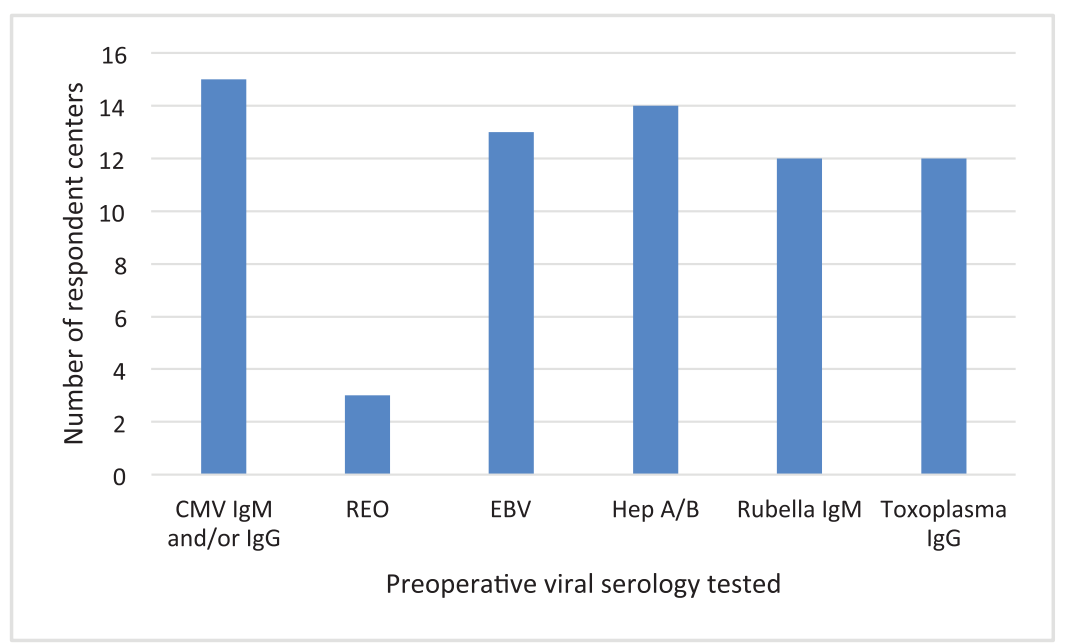

Fig. 2 Distribution of preoperative viruses tested. CMV, cytomegalovirus; Hep; hepatitis; IgG, immunoglobulin G; IgM, immunoglobulin M; REO, reovirus.

intravenous methylprednisolone in six centers. The modal dose of methylprednisolone was 3 to $5 \mathrm{mg} / \mathrm{kg} /$ day $(n=4)$. Prednisolone was given at a modal dose of 1 to $2 \mathrm{mg} / \mathrm{kg} /$ day $(n=2)$. Only three centers used high-dose prednisolone ( $\geq 4 \mathrm{mg} / \mathrm{kg} / \mathrm{day}$ ). Two centers used somewhat unusual steroids, i.e., oral dexamethasone $(0.3 \mathrm{mg} / \mathrm{kg} /$ day $)$ and rectal budesonide (no dose given).

Following a tapering regime, the steroids were prescribed for a median of 13 (4-24) weeks. At the 4 th week of followup, following an initial positive response to a KPE (i.e., pigmented stools and fading jaundice), only five centers would repeat a course of steroids if the infant's stools became pale, and the bilirubin started to rise. None would repeat more than one course.

\section{Choleretic Agents}

All centers used UDCA largely at $\geq 20 \mathrm{mg} / \mathrm{kg} / \mathrm{day}(n=9)$ or 15 to $19 \mathrm{mg} / \mathrm{kg} /$ day $(n=7)$. Phenobarbitone $(n=4)$ was given at a modal dose of 4 to $5 \mathrm{mg} / \mathrm{kg} /$ day $(n=2)$ and cholestyramine $(n=2)$ at 0.5 to 1 sachet/day $(n=2)$.

A single choleretic agent was used in 13 centers, most commonly UDCA. Dual choleretics were used in four centers -UDCA and phenobarbitone $(n=3)$ and UDCA and cholestyramine $(n=1)$.

\section{Antibiotics}

All centers used perioperative antibiotics. The commonest perioperative intravenous antibiotics were a combination of piperacillin-tazobactam (Tazocin) $(n=7)$ and gentamicin $(n=8)$. Thirteen centers subsequently prescribed oral prophylactic antibiotics, with median duration of 24 (452 ) weeks. Modal prophylactic antibiotic was co-trimoxazole $(n=7)$.

\section{Antivirals/Others}

If CMV-IgM was found to be positive, 7/15 (47\%) centers would treat with specific antiviral therapy, with the commonest being intravenous (IV) ganciclovir $(n=6)$. This was then changed to oral valganciclovir $(n=2)$. The median duration of treatment was 1 (1-3) months. Serial copies/ $\mathrm{mL}$ of CMV was measured $(n=2)$ to guide therapy duration. No centers used intravenous immunoglobulin (IVIG), traditional Chinese, or other herbal medicine.

\section{Outcome}

"Average" declared jaundice clearance at any point in time varied from 30 to $40 \%(n=3)$ to $>60 \%(n=5)$. The modal group was 41 to $50 \%(n=6)$. Those with the poorest outcomes were all from smaller centers ( $<5$ cases/year). Conversely, those with reported clearance rates of $>60 \%$ were from centers seeing $>5$ cases/year, though even those centers with $>15$ year did not necessarily have such high rates.

\section{Discussion}

Although BA is the most common cause of obstructive jaundice in infancy, its rarity limits high-quality evidencebased assessment of diagnostic tests and perioperative adjuvant regimens.

There is little evidence for a "gold-standard" test that confirms the diagnosis before laparotomy or laparoscopy. Most simply opt-for exclusion of more "medical" diagnoses such as $\alpha$-1-antitrypsin deficiency, Alagille syndrome, and familial cholestasis syndromes on biochemistry, genetic studies, and clinical features with more detailed imaging (typically ultrasound) failing to show any evidence of biliary tract dilatation as the prelude to (mini)laparotomy and confirmation on-table. Of the more invasive investigations, there are still European units who advocate liver biopsy and histology, but it is by no means widespread and its use anecdotally appears to be in decline. Only a single center opted for ERCP in the initial investigation strategy.

All centers in our survey currently perform an open KPE though one had been an initial advocate for the alternative, laparoscopic KPE during the 2000s. ${ }^{8,9}$ We tried to get an impression of more subtle details on the actual performance of the KPE operation by inquiring of depth of resection offering a relatively simple dissection leaving an ovoid of 
proximal remnant tissue as was originally described by Kasai compared with a more radical wider dissection where all the portal plate is denuded of remnant tissue in an around the branches of the right and left portal veins as suggested by Ohi, Kasai's successor in the Sendai unit. Most surgeons stated that they would do the more radical alternative. Interestingly, there was one surgeon who deliberately resected part of a liver segment in addition to the KPE, something without any real published support.

Postoperative prescription of steroids has been routine in some units since the 1990s to combat the proposed pathological role of inflammation. There have been two published placebo-controlled randomized trials, ${ }^{10,11}$ two prospective cohort-controlled open-label studies using high-dose steroids, ${ }^{12,13}$ and a multiplicity of smaller uncontrolled studies. ${ }^{14}$ Our experience at King's College Hospital would suggest benefit in terms of improved jaundice clearance and reduction in need for transplant with a high-dose regimen ( $\geq 4 \mathrm{mg} / \mathrm{kg} /$ day), ${ }^{10,13}$ and it is universal practice in the United Kingdom units. We have previously shown that the effect of steroids is age-dependent with an arbitrary limit of 70 days beyond which benefit is difficult to show. ${ }^{15}$ Though most European units do use steroids in one form or another though there appears to be no agreement on choice of steroids (though oral prednisone was the commonest) on drug dosage or on duration. Intravenous methylprednisolone is relatively common, but seemingly followed by a low-dose of prednisolone/prednisone for rather an extended period of approximately 12 to 13 weeks.

UDCA is a hydrophilic bile acid making up only a small (1$4 \%$ ) proportion of total the bile acid pool in humans. It is hypothesized that if one can increase this proportion, there will be a reduction in more "toxic" endogenous bile acids and therefore better protection of native hepatocytes and cholangiocytes. ${ }^{16}$ It has trial-proven efficacy in some adult cholestatic diseases, e.g., primary biliary cirrhosis and pediatric liver dysfunction of cystic fibrosis and perhaps prolonged parental nutrition associated jaundice, ${ }^{17}$ and there is single crossover trial showing improvement in liver biochemistry in children with stable post-KPE BA. ${ }^{18}$ Despite scarcity of evidence in BA and in this age-group, use of oral UDCA was universal in our survey. By contrast, older agents also advocated at one time as protective of hepatocyte function (e.g., phenobarbitone) or able to reduce the bile acid pool by intestinal sequestration (e.g., cholestyramine) have become unfashionable. $^{19}$

Postoperative cholangitis is common, bacterial in origin and various antibiotic regimens have been utilized to try and aid in its prevention. ${ }^{20}$ Still there are only four trials, but only one of them was randomized and included a placebo group. ${ }^{21,22}$ Evidence for continuation beyond the early postoperative period is therefore weak, and there is not prima facie evidence that long periods of oral offers any level of greater protection. ${ }^{22}$ Nonetheless immediate postoperative use of intravenous antibiotic was universal using agents, such as gentamicin and piperacillin/tazobactam (Tazocin). Most units also continue an oral antibiotic for long periods (6 months + ) with the commonest one being the venerable combination sulfamethoxazole/trimethoprim (Septrin).

A relatively new addition to the post-KPE protocol appears to be specific antiviral therapy. We and others have suggested that CMV IgM + ve BA is a distinct variant with a worse prognosis. ${ }^{7,23}$ Some centers do not routinely test for CMV serology, and many centers could not give any real idea of the percentage affected in their referral case-mix. Still just over half of those who do measure CMV serology would treat patients found to be IgM positive or with high viral loads.

Both intravenous ganciclovir and more recently its oral prodrug valganciclovir have been shown to reduce progression of congenital CMV disease affecting children's nervous systems. ${ }^{24}$ However, the published literature on its use in CMVIgM positive BA patients is small and involves only a handful of cases. ${ }^{25}$ Our unpublished experience with both antiviral agents seems to suggest that either is efficacious in improving outcome in this select group. Nonetheless some European authors would contest the actual influence of being "viral positive" (using an extended panel of viruses, not just CMV) as they could not show any difference in outcome. ${ }^{26}$ Still, native liver survival was relatively poor in both groups.

IVIG is an established treatment in multiple immunerelated inflammatory conditions and has been used to reduce inflammatory cytokines and increase anti-inflammatory $\mathrm{T}$ helper cells. In North America, a study of IVIG is currently underway (Post Portoenterostomy in Infants with Biliary Atresia [PRIME]). This was not used in any European center. Finally, traditional Chinese medicine Inchin-ko-to, ${ }^{27}$ Artemisia derivatives, and glycyrrhizin have been reported in the Japanese and Chinese literature and are currently widely prescribed there. Actual evidence is minimal though and they have yet to be used in Europe in any systematic fashion.

This survey can only report self-declared outcome statistics, but even so the range was wide with some centers suggesting clearance rates of $<40 \%$ despite very reasonable average ages at KPE. Published contemporary European outcomes ( - Table 1$)^{20,28-30}$ do show significant variation from

Table 1 Recent national studies of outcome in biliary atresia

\begin{tabular}{|l|l|l|l|l|l|}
\hline \multicolumn{1}{|l|}{} & $n$ & Period & Age at surgery & Clearance of jaundice \\
\hline National series & 439 & $1999-2009$ & 54 days & $55 \%$ \\
\hline England and Wales & 148 & $2005-2016$ & 64 days & $64 \%$ & $38 \%$ \\
\hline Finland, Sweden, Norway, and Denmark & 104 & $1998-2008$ & 59 days & $36 \%$ \\
\hline The Netherlands & 339 & $2003-2009$ & 59 days & \\
\hline France &
\end{tabular}


a reported clearance rate of $36 \%$ in France to one of $64 \%$ in the Nordic countries. Our survey reflects this variation. Of note, six of our survey centers were in "centralized" units in their respective countries.

We report the first survey of post-Kasai management from across Europe. Practice surveys are an effective tool for data gathering on the views, practice, and management of pediatric surgeons, particularly for rare conditions. However, surveys have obvious and known limitations. The reliability depends on several factors: answers may not be honest and accurate; by responding, there is an intrinsic selection bias (non-respondents may have answered differently), and options may be interpreted differently by different respondents from the same center. ${ }^{31}$

\section{Conclusion}

In conclusion, we have shown that there is a wide variability of postoperative adjuvant regimens in use across Europe. This reflects the multiplicity of languages and cultures within Europe, but is probably not the best environment for progress and achievement of the best results following KPE. Actual centralization requires political will, but adoption of common protocols does not. We would suggest adoption of more consistent guidelines across institutional and national boundaries. Self-reported outcomes also appear to be highly variable, and it is not obvious why this should be so. The creation of national prospective databases should be encouraged to help to identify areas where improvements could be made and areas of weakness identified.

\section{Conflict of Interest}

None.

\section{Acknowledgments}

The authors are thankful to the following respondents: Evelyn Peng Ong, Naved Alizai (United Kingdom); Jan Hulscher (the Netherlands); Dominique Debray, Christophe Chardot, and Florent Guerin (Paris); Filippo Parolini, Ernesto La Pergola, Daniele Alberti, Mario Lima, and Chiara Grimaldi (Italy); Claus Petersen and Udo Rolle (Germany); Ragnhild Emblem and Kristin Bjørnland (Norway); Jim Wilde and Barbara Wildhaber (Switzerland); Gonca Topuzlu Tekant (Turkey); Nina Qvist and Lars Søndergaard Johansen (Denmark); Nicholas Pachtner (Austria); Dani Arbell (Israel) and Alexander Passalides (Greece).

\section{References}

1 Hsiao C-H, Chang M-H, Chen H-L, et al; Taiwan Infant Stool Color Card Study Group. Universal screening for biliary atresia using an infant stool color card in Taiwan. Hepatology 2008;47(04): 1233-1240

2 Nio M. Japanese Biliary Atresia Registry. Pediatr Surg Int 2017;33 (12):1319-1325

3 Fischler B, Haglund B, Hjern A. A population-based study on the incidence and possible pre- and perinatal etiologic risk factors of biliary atresia. J Pediatr 2002;141(02):217-222
4 Wildhaber BE, Majno P, Mayr J, et al. Biliary atresia: Swiss national study, 1994-2004. J Pediatr Gastroenterol Nutr 2008;46(03): 299-307

5 Livesey E, Cortina Borja M, Sharif K, et al. Epidemiology of biliary atresia in England and Wales (1999-2006). Arch Dis Child Fetal Neonatal Ed 2009;94(06):F451-F455

6 Chardot C, Carton M, Spire-Bendelac N, Le Pommelet C, Golmard $\mathrm{JL}$, Auvert B. Epidemiology of biliary atresia in France: a national study 1986-96. J Hepatol 1999;31(06):1006-1013

7 Zani A, Quaglia A, Hadzić N, Zuckerman M, Davenport M. Cytomegalovirus-associated biliary atresia: an aetiological and prognostic subgroup. J Pediatr Surg 2015;50(10):1739-1745

8 Ure BM, Kuebler JF, Schukfeh N, Engelmann C, Dingemann J, Petersen C. Survival with the native liver after laparoscopic versus conventional Kasai portoenterostomy in infants with biliary atresia: a prospective trial. Ann Surg 2011;253(04):826-830

9 Oetzmann von Sochaczewski C, Petersen C, Ure BM, et al. Laparoscopic versus conventional Kasai portoenterostomy does not facilitate subsequent liver transplantation in infants with biliary atresia. J Laparoendosc Adv Surg Tech A 2012;22(04):408-411

10 Davenport M, Stringer MD, Tizzard SA, McClean P, Mieli-Vergani G, Hadzic N. Randomized, double-blind, placebo-controlled trial of corticosteroids after Kasai portoenterostomy for biliary atresia. Hepatology 2007;46(06):1821-1827

11 Bezerra JA, Spino C, Magee JC, et al; Childhood Liver Disease Research and Education Network (ChiLDREN). Use of corticosteroids after hepatoportoenterostomy for bile drainage in infants with biliary atresia: the START randomized clinical trial. JAMA 2014;311(17):1750-1759

12 Petersen C, Harder D, Melter M, et al. Postoperative high-dose steroids do not improve mid-term survival with native liver in biliary atresia. Am J Gastroenterol 2008;103(03):712-719

13 Davenport M, Parsons C, Tizzard S, Hadzic N. Steroids in biliary atresia: single surgeon, single centre, prospective study. J Hepatol 2013;59(05):1054-1058

14 Chen Y, Nah SA, Chiang L, Krishnaswamy G, Low Y. Postoperative steroid therapy for biliary atresia: Systematic review and metaanalysis. J Pediatr Surg 2015;50(09):1590-1594

15 Tyraskis A, Davenport M. Steroids after the Kasai procedure for biliary atresia: the effect of age at Kasai portoenterostomy. Pediatr Surg Int 2016;32(03):193-200

16 Yamashiro Y, Ohtsuka Y, Shimizu T, et al. Effects of ursodeoxycholic acid treatment on essential fatty acid deficiency in patients with biliary atresia. J Pediatr Surg 1994;29(03):425-428

17 Mousa HS, Lleo A, Invernizzi P, Bowlus CL, Gershwin ME. Advances in pharmacotherapy for primary biliary cirrhosis. Expert Opin Pharmacother 2015;16(05):633-643

18 Willot S, Uhlen S, Michaud L, et al. Effect of ursodeoxycholic acid on liver function in children after successful surgery for biliary atresia. Pediatrics 2008;122(06):e1236-e1241

19 Vajro P, Couturier M, Lemonnier F, Odièvre M. Effects of postoperative cholestyramine and phenobarbital administration on bile flow restoration in infants with extrahepatic biliary atresia. J Pediatr Surg 1986;21(04):362-365

20 de Vries W, de Langen ZJ, Groen H, et al; Netherlands Study Group of Biliary Atresia and Registry (NeSBAR). Biliary atresia in the Netherlands: outcome of patients diagnosed between 1987 and 2008. J Pediatr 2012;160(04):638-644

21 Bu L-N, Chen H-L, Chang C-J, et al. Prophylactic oral antibiotics in prevention of recurrent cholangitis after the Kasai portoenterostomy. J Pediatr Surg 2003;38(04):590-593

22 Decharun K, Leys CM, West KW, Finnell SME. Prophylactic antibiotics for prevention of cholangitis in patients with biliary atresia status post-Kasai portoenterostomy: a systematic review. Clin Pediatr (Phila) 2016;55(01):66-72

23 Shen C, Zheng S, Wang W, Xiao X-M. Relationship between prognosis of biliary atresia and infection of cytomegalovirus. World J Pediatr 2008;4(02):123-126 
6 Adjuvant Therapy Post-Kasai for Biliary Atresia Wong, Davenport

24 Kimberlin DW, Jester PM, Sánchez PJ, et al; National Institute of Allergy and Infectious Diseases Collaborative Antiviral Study Group. Valganciclovir for symptomatic congenital cytomegalovirus disease. N Engl J Med 2015;372(10):933-943

25 Fischler B, Svensson JF, Nemeth A. Early cytomegalovirus infection and the long-term outcome of biliary atresia. Acta Paediatr 2009; 98(10):1600-1602

26 Schukfeh N, Al-Gamrah A, Petersen C, Kuebler JF. Detection of hepatotropic viruses has no impact on the prognosis after Kasai procedure. J Pediatr Surg 2012;47(10):1828-1832

27 Tamura T, Kobayashi H, Yamataka A, Lane GJ, Koga H, Miyano T. Inchin-ko-to prevents medium-term liver fibrosis in postoperative biliary atresia patients. Pediatr Surg Int 2007;23(04):343-347
28 Chardot C, Buet C, Serinet MO, et al. Improving outcomes of biliary atresia: French national series 1986-2009. J Hepatol 2013;58(06): 1209-1217

29 Davenport M, Ong E, Sharif K, et al. Biliary atresia in England and Wales: results of centralization and new benchmark. J Pediatr Surg 2011;46(09):1689-1694

30 Pakarinen MP, Johansen LS, Svensson JF, et al; Nordic Pediatric Surgery Study Consortium. Outcomes of biliary atresia in the Nordic countries - a multicenter study of 158 patients during 2005-2016. J Pediatr Surg 2018;53(08): 1509-1515

31 Zani A, Zani-Ruttenstock E, Eaton S, Pierro A. The value of surveys in pediatric surgery. Eur J Pediatr Surg 2015;25(06):500-503 